\title{
Massive xanthomatosis and altered composition of atherosclerotic lesions in hyperlipidemic mice lacking acyl CoA:cholesterol acyltransferase 1
}

\author{
Michel Accad, ${ }^{1,2,3}$ Steven J. Smith, ${ }^{1,3}$ Dale L. Newland, ${ }^{1}$ David A. Sanan, ${ }^{1}$ Lloyd E. King, Jr., ${ }^{4}$ \\ MacRae F. Linton, ${ }^{5}$ Sergio Fazio, ${ }^{5}$ and Robert V. Farese, Jr. 1,2,3 \\ ${ }^{1}$ Gladstone Institute of Cardiovascular Disease, San Francisco, California 94141, USA \\ ${ }^{2}$ Department of Medicine, and \\ ${ }^{3}$ Cardiovascular Research Institute, University of California at San Francisco, San Francisco, California 94143, USA \\ ${ }^{4}$ Department of Dermatology, and \\ ${ }^{5}$ Department of Medicine, Vanderbilt University Medical Center, Nashville, Tennessee 37232, USA \\ Address correspondence to: Robert V. Farese, Jr., Gladstone Institute of Cardiovascular Disease, PO Box 419100, San Francisco, \\ California 94141-9100, USA. Phone: (415) 826-7500; Fax: (415) 285-5632; E-mail: bfarese@gladstone.ucsf.edu.
}

Received for publication November 30, 1999, and accepted in revised form February 7, 2000.

\begin{abstract}
Inhibitors of acyl CoA:cholesterol acyltransferase (ACAT) have attracted considerable interest as a potential treatment for atherosclerosis. Currently available inhibitors probably act nonselectively against the two known ACATs. One of these enzymes, ACAT1, is highly expressed in macrophages in atherosclerotic lesions, where it contributes to foam-cell formation. In this study, we examined the effects of selective ACAT1 deficiency in two mouse models of atherosclerosis. In the setting of severe hypercholesterolemia caused by deficiency in apoE or the LDL receptor (LDLR), total ACAT1 deficiency led to marked alterations in cholesterol homeostasis and extensive deposition of unesterified cholesterol in the skin and brain. Bone marrow transplantation experiments demonstrated that ACAT1 deficiency in macrophages was sufficient to cause dermal xanthomas in hyperlipidemic LDLR-deficient mice. ACAT1 deficiency did not prevent the development of atherosclerotic lesions in either apoE-deficient or LDLR-deficient mice, despite causing relatively lower serum cholesterol levels. However, the lesions in ACAT1-deficient mice were atypical in composition, with reduced amounts of neutral lipids and a paucity of macrophages in advanced lesions. Although the latter findings may be associated with increased lesion stability, the marked alterations in cholesterol homeostasis indicate that selectively inhibiting ACAT1 in the setting of severe hyperlipidemia may have detrimental consequences.
\end{abstract}

J. Clin. Invest. 105:711-719 (2000).

\section{Introduction}

Intracellular cholesterol esterification, catalyzed by the microsomal enzyme acyl CoA:cholesterol acyltransferase (ACAT) (1-3), has long been suspected of playing an important role in atherosclerosis development. ACAT synthesizes cholesterol esters that are incorporated into lipoproteins and secreted from the intestine and liver, and thus ACAT activity may modulate plasma cholesterol levels, an important risk factor for atherosclerosis. More directly, ACAT catalyzes the synthesis of cholesterol esters that accumulate in macrophages (4), thereby promoting macrophage foam-cell formation in the arterial wall, a hallmark of early atherosclerotic lesions.

Because of the potential role of ACAT in atherosclerosis, numerous ACAT inhibitors have been developed as possible therapeutic drugs (5-8). In many studies in animals, these agents both exerted hypolipidemic effects and reduced atherosclerosis (9-11). In some studies in cholesterol-fed rabbits, however, ACAT inhibitors reduced atherosclerosis without lowering plasma cholesterol levels $(12,13)$. Additionally, in cholesterol-fed hamsters, the inhibitor CI-1011 reduced lesion development more than expected from the amount of cholesterol lowering (14). These findings suggest that ACAT inhibitors reduce atherosclerosis by directly inhibiting cholesterol esterification in macrophage foam cells in the arterial wall. However, it is unknown whether cholesterol ester synthesis in macrophage foam cells by ACAT is beneficial, by limiting the toxicity of cholesterol-rich lipoproteins in the subendothelial space, or detrimental, by contributing to an inflammatory process in atherosclerotic lesions.

Current ACAT inhibitors were developed before the recent findings that two mammalian ACAT genes exist (15-18) (the mouse ACAT genes have been designated Soat 1 and Soat2). ACAT1 is expressed in many tissues (15); in mice, the highest expression levels are in macrophages, adrenal glands, dermal sebaceous glands, and preputial glands (19). ACAT1 is also expressed in atherosclerotic lesions $(19,20)$. ACAT2, however, is expressed only in the liver and intestine (16-18). By expressing the two mouse ACAT genes independently in insect cells, we recently demonstrated that the two enzymes differ in their sensitivity to specific ACAT 
inhibitors (16), although none of the inhibitors tested were selective for either ACAT. Nevertheless, these studies suggest that pharmacological agents potentially could be designed to inhibit specific ACAT enzymes.

To investigate the potential value of selective ACAT1 inhibition, we have used a mouse model of ACAT1 deficiency (21). ACAT1-deficient (ACAT1 ${ }^{-/}$) mice have
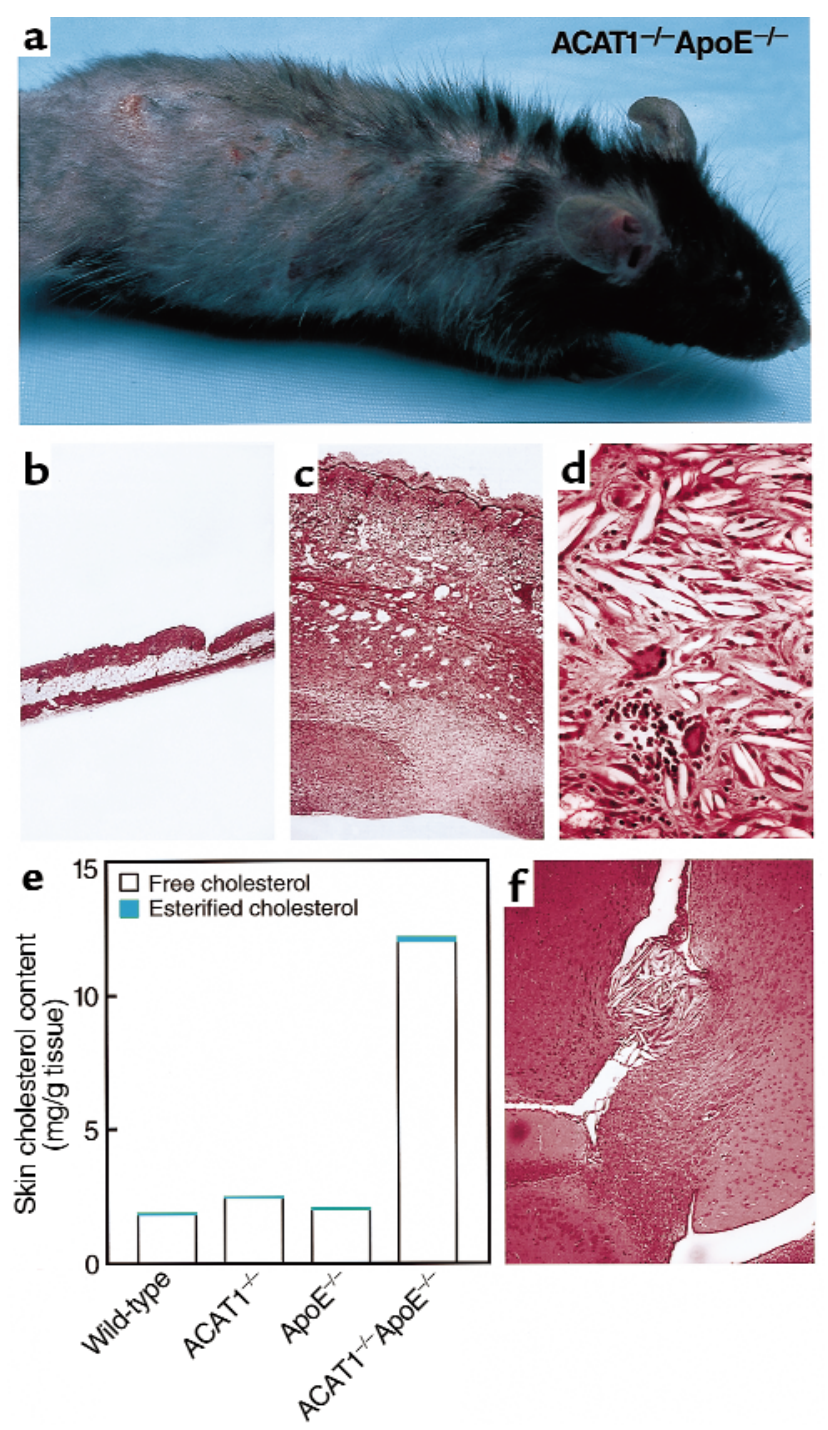

Figure 1

Severe xanthomatosis in ACAT1-/-ApoE-/- mice. (a) Four-month-old ACAT1 $1^{-/-} \mathrm{ApoE}^{-/-}$mouse fed a chow diet. Note the diffuse hair loss and excoriations due to pruritic skin lesions. (b-d) $\mathrm{H} \& \mathrm{E}$-stained sections of skin from 4-month-old, chow-fed ACAT1 ${ }^{+/+} \mathrm{ApoE}^{-/-}(\mathbf{b}, \times 40)$ and ACAT1 $1^{-1-} \mathrm{ApoE}^{-/-}(\mathbf{c}, \times 40 ; \mathbf{d}, \times 100)$ mice. Marked thickening of skin was observed in ACAT1-/-ApoE E $^{-/-}$mice (c), with an intense inflammatory reaction disrupting the subcutaneous fat layer and muscularis carnosus. Severe fibrosis was present in the deeper layers of the skin. Higher magnification of the reticular dermis (d) revealed numerous cholesterol clefts and a pleomorphic inflammatory infiltrate that included multinucleated giant cells, neutrophils, mononuclear cells, and plasma cells. (e) Skin cholesterol content of 4-month-old, chow-fed mice $(n=2$ in each group). (f) H\&E-stained section of brain from ACAT1 $1^{-1-} \mathrm{ApoE} \mathrm{E}^{-/-}$ mouse fed a Western diet for 3 months. Note the free cholesterol crystal deposits and inflammatory infiltrate near the choroid plexus. greatly reduced cholesterol ester levels in the adrenal cortex and in cultured peritoneal macrophages. However, their hepatic cholesterol esterification activity, intestinal cholesterol absorption, and plasma cholesterol levels (in 129/Sv inbred ACAT1 ${ }^{-/}$mice [R.V. Farese, Jr., and E. Sande, unpublished observations]) are normal. Because of their diminished foam-cell formation in macrophages and their unaltered plasma cholesterol levels, ACAT1-/- mice are ideally suited for testing the hypothesis that ACAT1 deficiency in arterial macrophages results in decreased atherosclerosis. In this study, we tested this hypothesis by crossing ACAT1 ${ }^{-/}$mice with two mutant mouse strains that are susceptible to atherosclerosis: apoE-deficient $\left(\mathrm{ApoE}^{-/-}\right)$ mice and $\mathrm{LDL}$ receptor-deficient $\left(\mathrm{LDLR}^{--}\right)$mice.

\section{Methods}

Mice. ACAT1/- mice (21) were crossed with $\mathrm{ApoE}^{-/-}$ mice (22) to generate ACAT1 $1^{-/-} \mathrm{ApoE}^{-/-}$mice and $\mathrm{ACAT}^{+/+} \mathrm{ApoE}^{-/-}$controls. The genetic background was mixed from C57BL/6J and $129 / \mathrm{Sv}$ inbred strains ( $\sim 63 \% \mathrm{C} 57 \mathrm{BL} / 6 \mathrm{~J})$. ACAT $1^{-/-}$mice were also crossed with $\mathrm{LDLR}^{-/-}$mice to generate ACAT1//-LDLR ${ }^{-/-}$mice and $\mathrm{ACAT} 1^{+/+} \mathrm{LDLR}^{-/-}$controls. The genetic background for these mice was approximately 99\% C57BL/6J. Genotyping was done by PCR for ACAT1 (21) and LDLR (23) and by Southern blotting for apoE (22). Mice were housed in a pathogen-free facility (12-hour dark and light cycles) and had free access to food and water. Mice were weaned onto diets at 4 weeks of age.

Diets. Excluding the bone marrow transplantation experiment, three diets were used: rodent chow (Ralston Purina, St. Louis, Missouri, USA), a customized high-fat Western diet containing 21\% (wt/wt) anhydrous milk fat and $0.15 \%$ (wt/wt) cholesterol (HarlanTeklad, Madison, Wisconsin, USA). and a synthetic atherogenic diet containing $7.5 \%$ (wt/wt) cocoa butter, $1.25 \%(\mathrm{wt} / \mathrm{wt}$ ) cholesterol, and $0.5 \%(\mathrm{wt} / \mathrm{wt}$ ) sodium cholate (ICN Biomedicals, Aurora, Ohio, USA).

Serum and tissue lipid analyses. Blood was obtained from the retroorbital plexus after a 4-hour fast. Colorimetric assays were used to measure total serum cholesterol (Spectrum kit; Abbott Diagnostics, Abbott Park, Illinois, USA) and free cholesterol (WAKO Chemicals, Neuss, Germany). Mouse serum was fractionated by fast-performance liquid chromatography (FPLC) through a Pharmacia Superose-6 column (Uppsala, Sweden), and fractions were assayed for total cholesterol concentrations. For tissue cholesterol measurements, mice were sacrificed by cervical dislocation and exsanguinated. Lipids were extracted from tissues, and their cholesterol ester and free cholesterol contents were measured by gas chromatography (24).

Histological analyses of tissues and atherosclerotic lesions. Tissues were fixed in neutral buffered formalin, dehydrated in ethanols, transitioned into xylene, and embedded in paraffin. Sections $(6 \mu \mathrm{m})$ were stained with hematoxylin and eosin (H\&E). Alternatively, to preserve and 
accentuate neutral lipids, tissues were stained in $2 \%$ osmium tetroxide in $0.1 \mathrm{M}$ phosphate buffer ( $\mathrm{pH} 7.4$ ), for 4-6 hours after formalin fixation. Tissues were dehydrated in a graded ethanol series, transitioned into propylene oxide, and embedded in epoxy resin (Polybed 812; Polysciences, Warrington, Pennsylvania, USA). Sections $(1 \mu \mathrm{m})$ were cut with a glass knife and stained in warm toluidine blue. Histological analysis was performed in 4-6 mice of each genotype.

For immunostaining, mouse tissues were perfusionfixed with 3\% paraformaldehyde in PBS ( $\mathrm{pH} 7.4)$. The hearts and aortic roots were dissected and fixed by immersion for 2 hours in the same fixative at $4{ }^{\circ} \mathrm{C}$, embedded in OCT, frozen in liquid nitrogen, and sectioned $(10 \mu \mathrm{m})$ with a cryostat microtome. Sections were immunostained with anti-mouse MOMA-2 rat mAb (BioSource International, Camarillo, California, USA). The primary antibody was detected by biotinylated sheep anti-rat IgG secondary antibody (Amersham Pharmacia Biotech, Piscataway, New Jersey, USA) followed by horseradish peroxidase- $Z$-avidin and the chromogen aminoethyl carbazole (Zymed, South San Francisco, California, USA). Hematoxylin was used as a counterstain. Smooth muscle cells were immunostained with a mouse $\mathrm{mAb}$ that recognizes human smooth muscle actin. This antibody was conjugated directly to horseradish peroxidase with a molecular stalk (EPOS/HRP; DAKO Corp., Carpinteria, California, USA), eliminating the need for a secondary antibody that could immunoreact with endogenous mouse IgG. Aminoethyl carbazole was used as the chromogen, and hematoxylin, as a counterstain.

Bone marrow transplantation experiment. Mice were fed a chow diet before transplantation. Bone marrow was collected from donor $\mathrm{ACAT}^{+/+} \mathrm{LDLR}^{-/}$or ACAT1 $1^{-/-} \mathrm{LDLR}^{-/-}$mice by flushing femurs and tibias with RPMI 1640 containing 2\% FBS and $5 \mathrm{U} / \mathrm{mL}$ heparin (Sigma Chemical Co., St. Louis, Missouri, USA) (25). Four hours before transplantation, 12 male 15 -week-old recipient $\mathrm{LDLR}^{-/-}$mice were lethally irradiated with 9 Gy from a cesium $\gamma$ source. Cells were counted, resuspended in RPMI $1640\left(5 \times 10^{6}\right.$ cells in $300 \mu \mathrm{L}$ ), and injected into the tail vein of $\mathrm{LDLR}^{-/-}$recipient mice (six mice received $\mathrm{ACAT}^{1+/+} \mathrm{LDLR}^{-/-}$marrow, and six mice received ACAT1 $1^{-/} \mathrm{LDLR}^{-/-}$marrow). A week before and two weeks after transplantation, neomycin $(100 \mathrm{mg} / \mathrm{L})$ and polymyxin B sulfate (10 $\mathrm{mg} / \mathrm{L}$ ) (both from Sigma Chemical Co.) in acidified water were administered to recipient mice. After transplantation, mice were fed a chow diet (PMI Feeds, Richmond, Indiana, USA) for four weeks, followed by an atherogenic butterfat diet (ICN Biomedicals) containing $19.5 \%$ fat, $1.25 \%$ cholesterol, and $0.5 \%$ cholic acid for ten weeks. Mice were then sacrificed, and 1-inch squares of abdominal or lesion area skin were excised, immersion-fixed in 4\% paraformaldehyde, embedded in paraffin, sectioned, and stained with H\&E. Alternatively, skin samples were embedded in OCT for cryosectioning and immunohistochemistry. For these experiments, sections were immersed in PBS ( $\mathrm{pH} 7.4$ ), and blocked with nonimmune goat serum before incubation for 30 minutes at $37^{\circ} \mathrm{C}$ with either a rabbit antiserum that recognizes the $\mathrm{NH}_{2}$-terminus of mouse ACAT1 (19) or a polyclonal rat antibody that detects the mouse monocyte-macrophage marker MOMA-2 (Accurate Chemical \& Scientific, Westbury, New York, USA). Sections were then incubated for 30 minutes at $37^{\circ} \mathrm{C}$ with biotinylated polyclonal antibodies that detect rabbit IgG or rat IgG (PharMingen, San Diego, California, USA), followed by avidin-biotin complex labeled with alkaline phosphatase (Vector Laboratories, Burlingame, California, USA). Reaction products were viewed with Fast Red TR/Naphthol AS-NX substrate (Sigma Chemical Co.). Hematoxylin was used as a counterstain. As a negative control, nonimmune rabbit or rat serum was used in the place of primary antibody.

Statistical analyses. Serum and tissue cholesterol levels are shown as mean \pm SD. Means were compared with the Mann-Whitney rank-sum test.

\section{Results}

Altered cholesterol homeostasis in $A C A T^{-/} A p o E^{-/-}$mice. ACAT1-/-ApoE ${ }^{-/-}$mice initially appeared healthy but developed severe skin lesions within months when they were fed a chow diet. By age 2-3 months, their fur appeared dull and they had pruritus. Several weeks later, hair loss and excoriations ensued (Figure 1a), which led to severe lesions necessitating euthanasia of the mice by age 5-6 months. Histological analysis of the skin

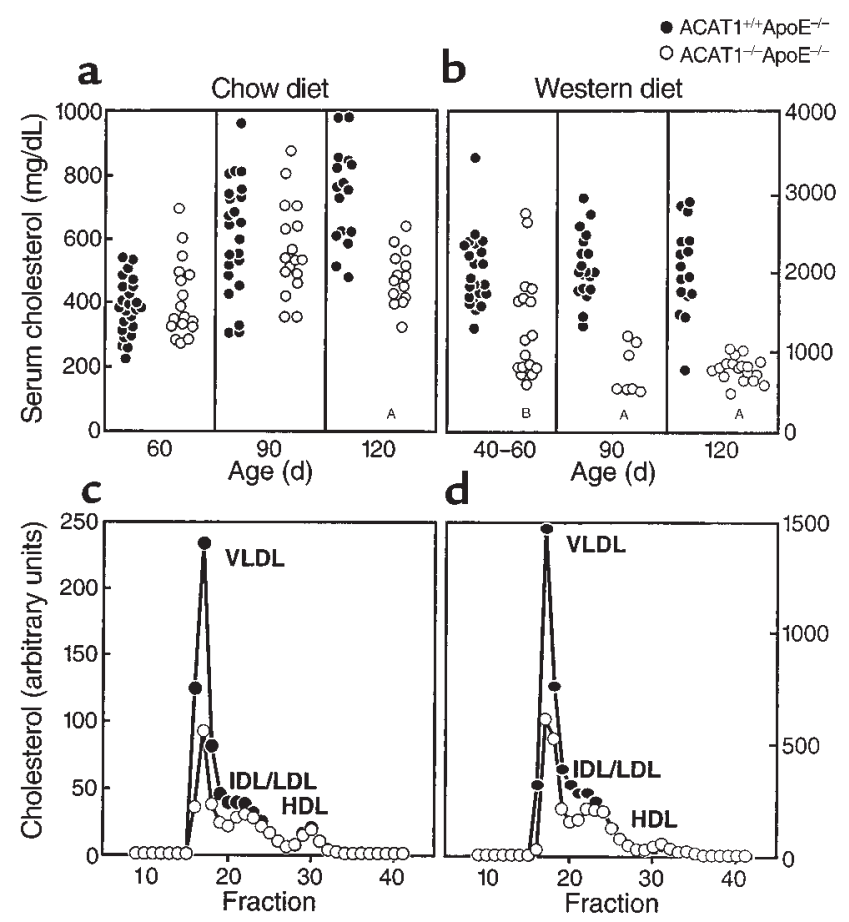

Figure 2

Relatively reduced serum cholesterol levels in ACAT1 $1^{-/-}$ApoE ${ }^{-/-}$mice compared with ACAT $1^{+/+} \mathrm{ApoE}^{-/-}$mice. Serum cholesterol levels in mice fed a chow diet (a) or a Western $\operatorname{diet}(\mathbf{b})$. (c and $\mathbf{d})$ Total cholesterol levels for pooled serum ( $n=5$ in each group) fractionated by FPLC. ${ }^{A} P<0.001,{ }^{B} P=0.002$. 

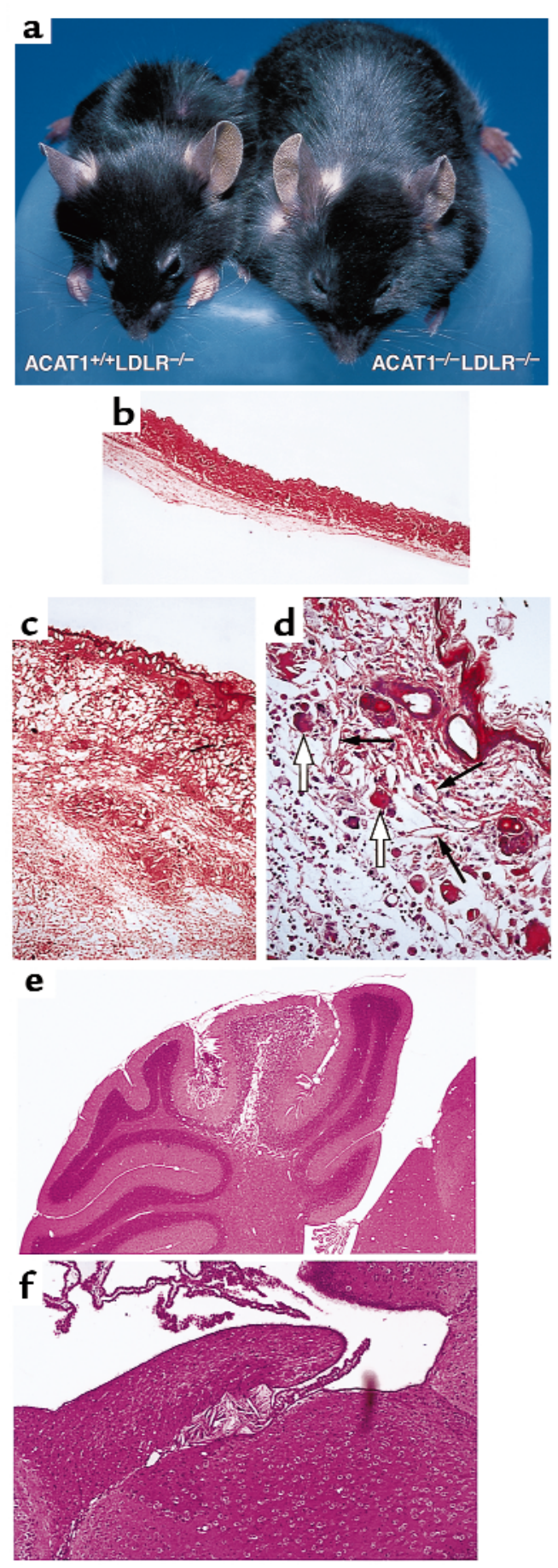

\section{Figure 3}

Severe xanthomatosis in ACAT1-/-LDLR ${ }^{-/-}$mice fed an atherogenic diet for 2 months. (a) Appearance of ACAT1-/-LDLR $/$ - mouse compared with a ACAT1 1/+'LDLR ${ }^{-/-}$littermate control. The marked skin thickening gives the ACAT1/-LDLR $/$ - mouse an obese appearance. (b-d) In H\&E-stained skin sections, no abnormalities were detected in ACAT $1^{+/+}$LDLR $^{-/-}$controls (b, $\left.\times 40\right)$; thickened skin and inflammation were present in ACAT1-/-LDLR ${ }^{-/-}$mice $(\mathbf{c}, \times 40 ; \mathbf{d}, \times 100)$. The marked skin thickening in ACAT1 $1 /-\mathrm{LDLR}^{-/-}$mice ( $\mathbf{c}$ and $\mathbf{d}$ ) was associated with dermal edema and a pleomorphic inflammatory infiltrate with cholesterol clefts (d, thin arrows) and multinucleated giant cells (d, open arrows). (e and $\mathbf{f}$ ) H\&E-stained sections of brain of ACAT1 $1 /-\mathrm{LDLR}^{-1-}$ mice demonstrating free cholesterol crystal deposits in the cerebellum $(\mathbf{e}, \times 10)$ and near the choroid plexus $(\mathbf{f}, \times 40)$. revealed marked thickening of the dermis (Figure 1, b and c) with diffuse needle-shaped lucencies indicative of cholesterol crystals (Figure 1d). The crystals appeared to be predominantly extracellular and were found in or below the reticular dermis. The free cholesterol content of the skin of chow-fed ACAT1 $1^{-/-} \mathrm{ApoE}^{-/-}$mice was 6- to 7 -fold higher than in control mice at age four months (Figure 1e). The papillary dermis appeared normal, as did the epidermis, except for marked hyperkeratosis at the surface. The cholesterol deposition was accompanied by a severe, pleomorphic inflammatory cell infiltrate with features of acute and chronic inflammation, including large numbers of neutrophils, numerous multinucleated giant cells with occasional intracellular cholesterol clefts, and frequent mononuclear cells, lymphocytes, and plasma cells (Figure 1d). The deeper layers of the dermis were characterized by a marked proliferation of fibroblasts, and pentachrome staining revealed extensive collagen deposition (data not shown). Skin abnormalities were not observed in $\mathrm{ACAT}^{+/+} \mathrm{ApoE}^{-/-}$or in $\mathrm{ACAT1}^{+/-} \mathrm{ApoE}^{-/-}$control mice. When the ACAT1 $1^{-/} \mathrm{ApoE}^{-/-}$mice were fed a Western diet, the skin changes occurred at an earlier age (detectable by $\sim 6$ weeks) and were more severe. By age 3-4 months, the skin covering the truncal region was essentially one massive cholesterol xanthoma.

Free cholesterol crystal deposits were also found in the brains of ACAT1 $1 /-\mathrm{ApoE}^{-/-}$mice fed a Western diet for three months (Figure 1f). The deposits occurred mostly near the choroid plexus. No cholesterol crystals were observed in brains of $\mathrm{ACAT}^{+/+} \mathrm{ApoE}^{-/-}$control mice. No overt changes in behavior were noted in the ACAT1 $1^{-/} \mathrm{ApoE}^{-/-}$mice, although formal neurologic testing was not performed. Other organs, including the liver, kidneys, small intestine, muscle, lungs, spleen, and heart, appeared normal in ACAT1 $1^{-/} \mathrm{ApoE}^{-/-}$mice fed a chow or Western diet.

In chow-fed ACAT1-/-ApoE-/- and ACAT1 ${ }^{+/+} \mathrm{ApoE}^{-/-}$ mice, serum cholesterol levels were similar at ages 60 and 90 days (Figure 2a). By age 120 days, however, mean serum cholesterol levels were significantly lower in ACAT1 $1 /-\mathrm{ApoE}^{-/}$mice than in controls (480 $\pm 86 \mathrm{vs.}$ $738 \pm 151 \mathrm{mg} / \mathrm{dL} ; P<0.001)$. The disparity in cholesterol levels was more marked and occurred at an earlier age in mice fed the Western diet (Figure 2b). By age 40-60 days, mean serum cholesterol levels were significantly lower in $\mathrm{ACAT}^{-/-} \mathrm{ApoE}^{-/-}$mice than in $\mathrm{ACAT}^{+/+} \mathrm{ApoE}^{-/-}$controls $(1,348 \pm 715$ vs. $2,024 \pm 472$ $\mathrm{mg} / \mathrm{dL} ; P=0.002)$. Serum cholesterol levels in the double-knockout mice were further reduced at age 90 days (777 \pm 309 vs. $2,091 \pm 425 \mathrm{mg} / \mathrm{dL}$ for controls; $P<$ $0.001)$ and age 120 days (793 \pm 142 vs. $2,054 \pm 575$ $\mathrm{mg} / \mathrm{dL}$ for controls; $P<0.001)$. The weights of ACAT1 $1 /-\mathrm{ApoE}^{-/-}$and $\mathrm{ACAT1} 1^{+/} \mathrm{ApoE}^{-/-}$mice fed a high-fat diet for 3 months were similar (30.9 \pm 3.7 vs. $29.3 \pm 4 \mathrm{~g}$ ), making it unlikely that poor nutrient intake was responsible for the relatively decreased serum cholesterol levels in $\mathrm{ACAT}^{-/} \mathrm{ApoE}^{-/}$mice.

The relatively lower serum cholesterol levels in the 
a

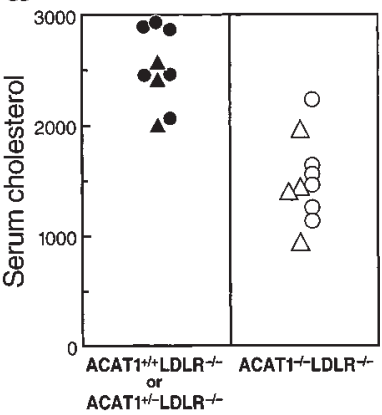

b

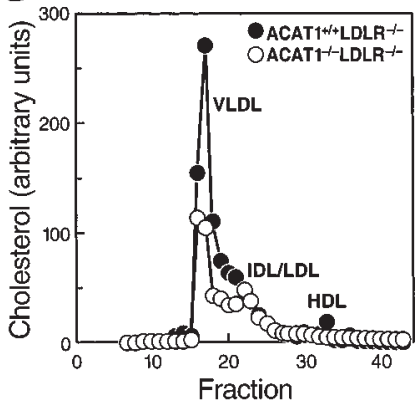

Figure 4

Relatively reduced serum cholesterol levels in ACAT1-/-LDLR ${ }^{-/-}$mice compared with ACAT1 $1^{+/+} \mathrm{LDLR}^{-/-}$mice. (a) Serum cholesterol levels in mice fed an atherogenic diet for 2 months. (b) Total cholesterol levels in pooled serum ( $n=5$ in each group) fractionated by FPLC. VLDL, very low density lipoproteins; IDL, intermediate density lipoproteins; LDL, low density lipoproteins; HDL, high density lipoproteins. Triangles represent values for male mice and circles represent values for females.

ACAT1 $1-/ \mathrm{ApoE}^{-/-}$mice resulted from reduced amounts in the VLDL and IDL fractions (Figure 2, $\mathrm{c}$ and d). The proportion of free cholesterol in the serum was slightly higher in ACAT1 $1^{-/} \mathrm{ApoE}^{-/-}$mice than in $\mathrm{ACAT1}^{+/+} \mathrm{ApoE}^{-/}$ mice ( $31.6 \pm 1.6 \%$ vs. $27.6 \pm 1.8 \% ; P<0.006)$.

Altered cholesterol homeostasis in ACAT1 $1^{-/-} L D L R^{-/-}$mice. ACAT1-/-LDLR ${ }^{-/-}$mice fed an atherogenic diet, but not those fed a chow diet, also developed diffuse, massive thickening of the skin compared with $\mathrm{ACAT1} 1^{+/+} \mathrm{LDLR}^{-/}$ control littermates (Figure 3, a-c). Skin thickening became apparent after the mice had consumed the atherogenic diet for 2 weeks. After 2 months on the diet, the mice had to be euthanized because of inanition due to compromised mobility. Unlike the findings in the $\mathrm{ACAT1}^{-/-} \mathrm{ApoE}^{-/-}$mice, however, the skin disease in ACAT1 $1-$ LDLR $^{-/-}$mice did not appear to be pruritic, hair loss was scant, and skin excoriations did not occur. On dissection, the skin appeared edematous. Histological analysis revealed diffuse deposition of cholesterol crystals in the dermis (Figure 3, c and d). In contrast to what was found in ACAT1 $1-\mathrm{ApoE}^{-/-}$mice, the crystals occurred in both the reticular and papillary dermis. A severe, pleomorphic inflammatory reaction accompanied the cholesterol deposition, but its features were different from those in the $\mathrm{ACAT} 1^{-/} \mathrm{ApoE}^{-/-}$mice. In ACAT1 $1 /-\mathrm{LDLR}^{-/-}$mice, the histological appearance was characteristic of edema, and the inflammatory cells were predominantly macrophages, lymphocytes, and plasma cells, with many fewer neutrophils than in ACAT1 $1^{-/} \mathrm{ApoE}^{-/-}$skin (Figure 3d).

Crystalline deposits were also found in the brains of all ACAT1-/-LDLR ${ }^{-/}$mice $(n=6)$ fed the atherogenic diet. The deposits occurred particularly near the choroid plexus and in the cerebellum (Figure 3, e and f). Only one of six ACAT1 $1^{+/} \mathrm{LDLR}^{-/}$control mice had small crystals near the choroid plexus. The brain cholesterol deposits might have contributed to the inani- tion displayed by ACAT1-/-LDLR-/- mice; however, formal neurologic testing to address this possibility was not performed. Cholesterol deposits were not observed in the liver, kidney, small intestine, muscle, lung, spleen, or heart of ACAT1 $1^{-/} \mathrm{LDLR}^{-/-}$mice.

Serum total cholesterol levels in mice fed the atherogenic diet for two months were lower in $\mathrm{ACAT1}^{-/} \mathrm{LDLR}^{-/}$ mice than in littermate $\mathrm{ACAT} 1^{+/+} \mathrm{LDLR}^{-/}$or

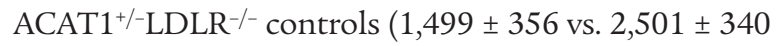
$\mathrm{mg} / \mathrm{dL} ; P<0.001$ ) (Figure 4a). The lower cholesterol levels resulted from reduced cholesterol levels in the VLDL and IDL fractions (Figure 4b).

Dermal xanthomatosis in LDLR-deficient mice with ACAT1-deficient bone marrow. To determine whether ACAT1 deficiency in skin macrophages contributed to the development of the dermal skin lesions, we transplanted marrow from ACAT1 $1^{-/} \mathrm{LDLR}^{-/}$mice or $\mathrm{ACAT}^{+/+} \mathrm{LDLR}^{-/-}$controls into $\mathrm{ACAT1} 1^{+/+} \mathrm{LDLR}^{-/}$ recipient mice. After transplantation, mice were fed an
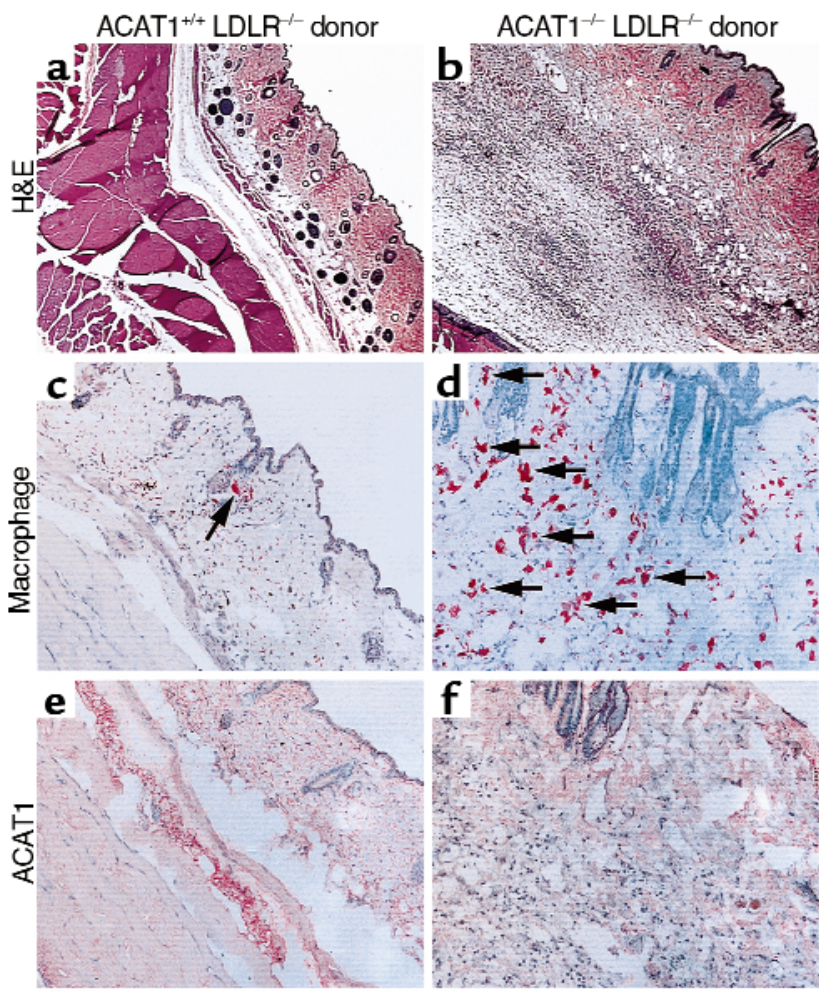

Figure 5

Dermal xanthomatosis in LDLR-deficient mice transplanted with marrow from ACAT1-/-LDLR $/$ - mice. Bone marrow was harvested from $\mathrm{ACAT1}^{+/+} \mathrm{LDLR}^{-/-}$or ACAT1 1/-LDLR ${ }^{-/-}$mice and transplanted into 15 -week-old LDLR-deficient recipients. Sections of skin were analyzed after 10 weeks of high-fat feeding. ( $\mathbf{a}$ and $\mathbf{b}$ ) Representative $\mathrm{H} \& \mathrm{E}$-stained sections of skin viewed at the same magnification $(\times 20)$. Note the marked thickening of the dermis in the mouse that received $\mathrm{ACAT1} 1^{-/-} \mathrm{LDLR}^{-/-}$marrow. (c and $\mathbf{d}$ ) Immunostaining for macrophages with MOMA-2 antiserum. Numerous macrophages (arrows) were observed in recipients of ACAT1-/-LDLR-/- marrow. (e and $\mathbf{f}$ ) Immunostaining for mouse ACAT1 with an ACAT1-specific antiserum. Specific staining for ACAT1 was observed in the dermis only in recipients of $A C A T 1^{+/+} \mathrm{LDLR}^{-/-}$marrow. 


\section{Figure 6}

Neutral lipid-poor atherosclerotic lesions in ACAT1-deficient mice. (a and b) Representative Oil-Red-Ostained aortic lesions from 6-monthold ACAT1 ${ }^{+/+} \mathrm{ApoE}^{-/-}$(a) and ACAT1 $1-\mathrm{ApoE}^{-/-}$(b) mice fed a chow diet. Note the pale appearance of the ACAT1-/-ApoE - $^{-/}$lesions due to a paucity of neutral lipids that stain with Oil Red O. (c and d) Representative sections of plastic-embedded, osmium tetroxide-stained aortic lesions from ACAT1 ${ }^{+/+} \mathrm{LDLR}^{-/-}$(c) and ACAT1 $1^{-/-} \mathrm{LDLR}^{-/-}$(d) mice fed an atherogenic diet for 2 months. Neutral lipid droplets (thin arrows) and numerous lucencies from cholesterol crystals (thick arrows) are apparent in the $\mathrm{ACAT} 1^{+/+} \mathrm{LDR}^{-/-}$lesion (c). ACAT1-/-LDLR ${ }^{-/-}$lesions were devoid of osmium tetroxide staining and had fewer cholesterol crystals than ACAT1 ${ }^{+/+}$LDLR $^{-/-}$lesions.
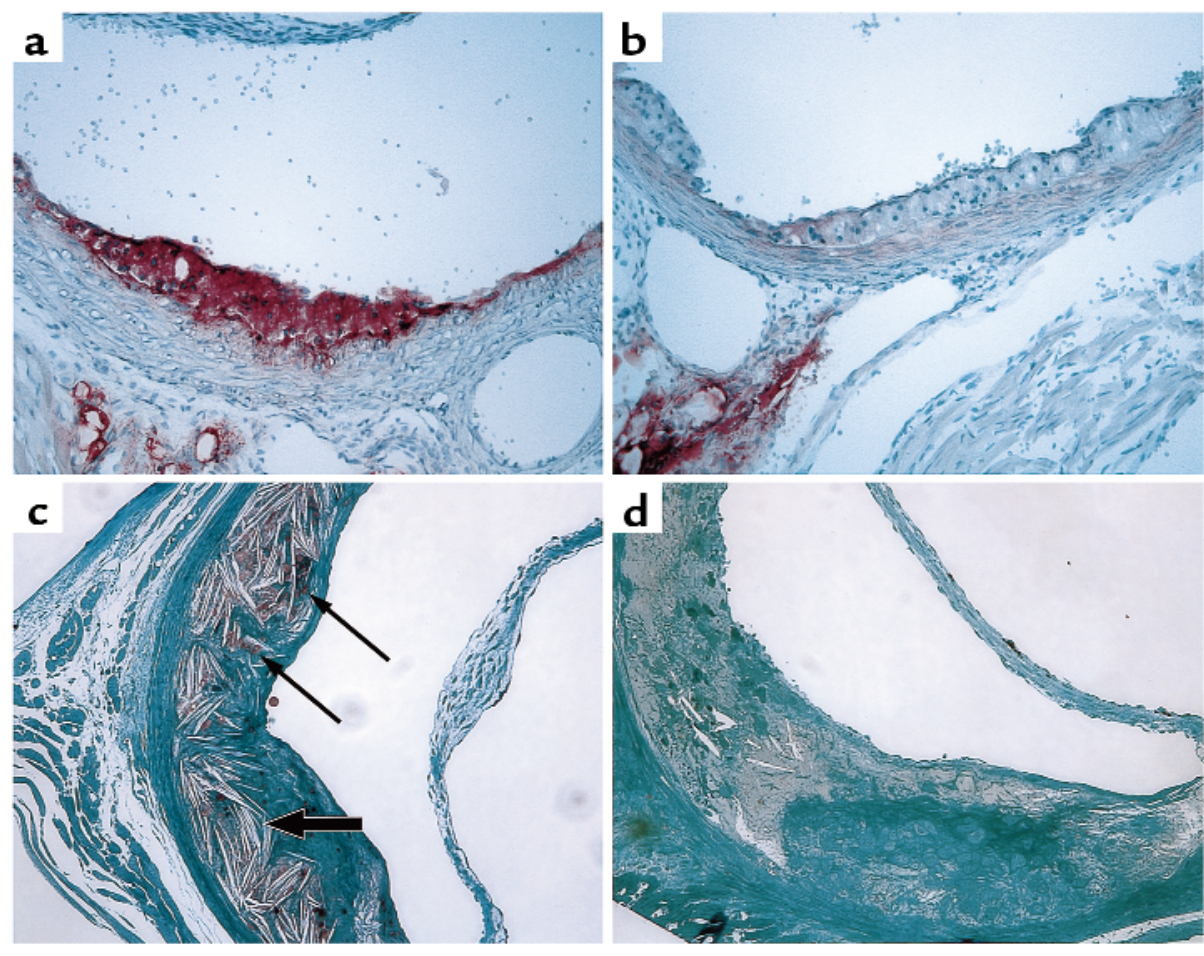

atherogenic high-fat diet for 10 weeks. By then, several recipients of ACAT1-deficient marrow appeared to have thickened skin. Histological analysis revealed marked thickening of the dermis in all recipients of ACAT1-/-LDLR ${ }^{-/}$marrow, whereas skin sections appeared normal in ACAT $1^{+/+} \mathrm{LDLR}^{-/}$recipients (Figure 5 , a and b). Needle-shaped lucencies indicating cholesterol crystals were observed at higher magnification (data not shown). Sections from recipients of ACAT1deficient marrow had many more macrophages than did sections from mice that received ACAT1-positive marrow (Figure 5, $\mathrm{c}$ and d), consistent with a marked inflammatory response. Staining of sections with an antiserum recognizing ACAT1 demonstrated that the repopulation of skin macrophages with cells from transplanted marrow was complete (Figure 5, e and f). Altered composition of atherosclerotic lesions in apoE-deficient mice or LDLR-deficient mice lacking ACAT1. Atherosclerotic lesions developed in both apoE-deficient and LDLR-deficient mice regardless of whether ACAT1 was present or deficient. Gross inspection of the aortas showed smaller lesions in ACAT1 $1^{-/-} \mathrm{ApoE}^{-/-}$mice than in $\mathrm{ACAT}^{+/+} \mathrm{ApoE}^{-/-}$controls fed a Western diet for 3 months; however, serum cholesterol levels were also much lower in $\mathrm{ACAT}^{-/-} \mathrm{ApoE}^{-/-}$mice, making it impossible to determine the specific effect of ACAT1 deficiency on lesion size. To circumvent this problem, we examined lesions in a subset of 90-day-old chowfed ACAT1 $1^{-/-} \mathrm{ApoE}^{-/-}$mice with cholesterol levels similar to those of ACAT1 ${ }^{+/+} \mathrm{ApoE}^{-/-}$controls (Figure 2a). However, in apoE knockout mice of that age, the small number of lesions and the high degree of variability in lesion size precluded a reliable quantitative analysis. Similarly, unmatched serum cholesterol levels in
$\mathrm{ACAT1}^{+/+} \mathrm{LDLR}^{-/-}$or ACAT1 1-/-LDLR ${ }^{-/-}$mice fed the atherogenic diet precluded quantitative analysis of atherosclerosis in this model. We therefore focused our analyses on the qualitative features of atherosclerotic lesions in ACAT1-deficient mice.

Proximal aortic sections (immediately distal to the sinus of Valsalva) were examined in apoE-deficient mice and in LDLR-deficient mice. Early, fatty streaktype lesions and more advanced, complex lesions were seen in both models in the presence and absence of ACAT1 (Figure 6). However, important differences in lesion composition were evident in ACAT1-deficient mice compared with their respective $\mathrm{ACAT} 1^{+/+}$controls. Oil-Red-O staining showed that the neutral lipid content of aortic lesions was markedly lower in both ACAT $1^{-/-} \mathrm{ApoE}^{-/-}$mice (compare Figure 6a with Figure 6b) and ACAT1-/-LDLR ${ }^{-/-}$mice (data not shown) than in controls. This difference was most striking in the early, fatty streak-type lesions, which are normally rich in macrophage-derived foam cells. Osmium tetroxide staining of more advanced lesions in ACAT1-/-LDLR-/mice confirmed the paucity of neutral lipids, and the sections also exhibited fewer cholesterol crystals than in lesions of ACAT1 ${ }^{+/+} \mathrm{LDLR}^{-/-}$mice (Figure 6, $\mathrm{c}$ and d).

In both apoE-deficient and LDLR-deficient mice fed high-fat diets, macrophage-specific immunostaining of aortic lesions was reduced in ACAT1-deficient mice compared with controls. In the advanced, complex lesions frequently observed in the ACAT1 $1^{+/} \mathrm{LDLR}^{-/-}$ mice fed the atherogenic diet, macrophage staining was present in necrotic core and subendothelial regions (Figure 7a). In contrast, macrophage staining was virtually absent in advanced lesions of ACAT1-deficient mice (Figure 7b). In the chow-fed apoE-deficient model, 
which was characterized by more early, fatty streak-type lesions, the reduction in macrophage staining in ACAT1-deficient lesions was also apparent although not as marked. Whereas ACAT1 ${ }^{+/+} \mathrm{ApoE}^{-/-}$lesions had macrophage staining throughout the lesions (Figure 7c), staining was reduced in ACAT1 $1^{-/-} \mathrm{ApoE}^{-/-}$lesions, particularly in the necrotic core regions (Figure $7 \mathrm{~d}$ ). However, macrophage staining was present in early, fatty-streak lesions of ACAT $1^{+/+} \mathrm{ApoE}^{-/-}$mice (Figure 7e). Immunostaining for smooth muscle cells in lesions showed no differences among the four genotypes of mice (data not shown).

\section{Discussion}

In this study, selective ACAT1 deficiency in the setting of severe hypercholesterolemia caused by apoE deficiency or LDLR deficiency led to markedly altered cholesterol homeostasis and extensive deposition of unesterified cholesterol in skin and brain. ACAT1 deficiency did not prevent the development of atherosclerotic lesions, despite causing relatively lower serum cholesterol levels. However, the lesions were atypical in composition, with reduced amounts of neutral lipids and a paucity of macrophages in the most advanced lesions. Although these changes could have beneficial effects on lesion stability, the marked alterations in cholesterol homeostasis demonstrate that selectively inhibiting ACAT1 in the setting of severe hyperlipidemia may have detrimental consequences.

The xanthomatosis in hyperlipidemic ACAT1-deficient mice was atypical in that it was caused by the deposition of unesterified cholesterol rather than cholesterol esters, which are found in typical xanthomas that occur in genetic disorders of cholesterol metabolism. The free cholesterol accumulation associated with ACAT1 deficiency occurred as crystals, which appeared to be extracellular and accompanied by a marked inflammatory response. Similar pathological findings have been reported in normolipidemic humans with diffuse cutaneous xanthomas $(26,27)$, although the etiology of these disorders remains uncertain.

The basic pathological process in the skin appeared to be similar in the setting of either apoE or LDLR deficiency, but the two models had different features. ApoE-deficient mice lacking ACAT1 had intense skin fibrosis and pruritus, whereas the LDLR-deficient mice had more edematous lesions. This suggests that the lack of apoE in the skin promotes a proinflammatory condition different from that promoted by LDLR deficiency. Inflammatory skin lesions have been described previously in both apoE-deficient mice and LDLR-deficient mice, but these changes were only observed microscopically after the mice had consumed an atherogenic diet for several months $(28,29)$.

ACAT1 $1^{-/} \mathrm{ApoE}^{-/-}$mice had more severe skin lesions when they were fed a high-fat diet and developed higher blood cholesterol levels than when they were fed a chow diet. Similarly, skin lesions were only apparent in ACAT1-/-LDLR ${ }^{-/}$mice with marked hypercholesterolemia due to the atherogenic diet and were not apparent when the mice were fed a chow diet, which resulted in only modest hypercholesterolemia. In both models, the deposition of unesterified cholesterol in the skin of ACAT1-deficient mice was accompanied by a relative reduction in serum cholesterol levels. We therefore hypothesize that the severe hyperlipidemia resulted in a high flux of cholesterol-containing lipoproteins through the skin, where perhaps reverse cholesterol transport mechanisms were overwhelmed.
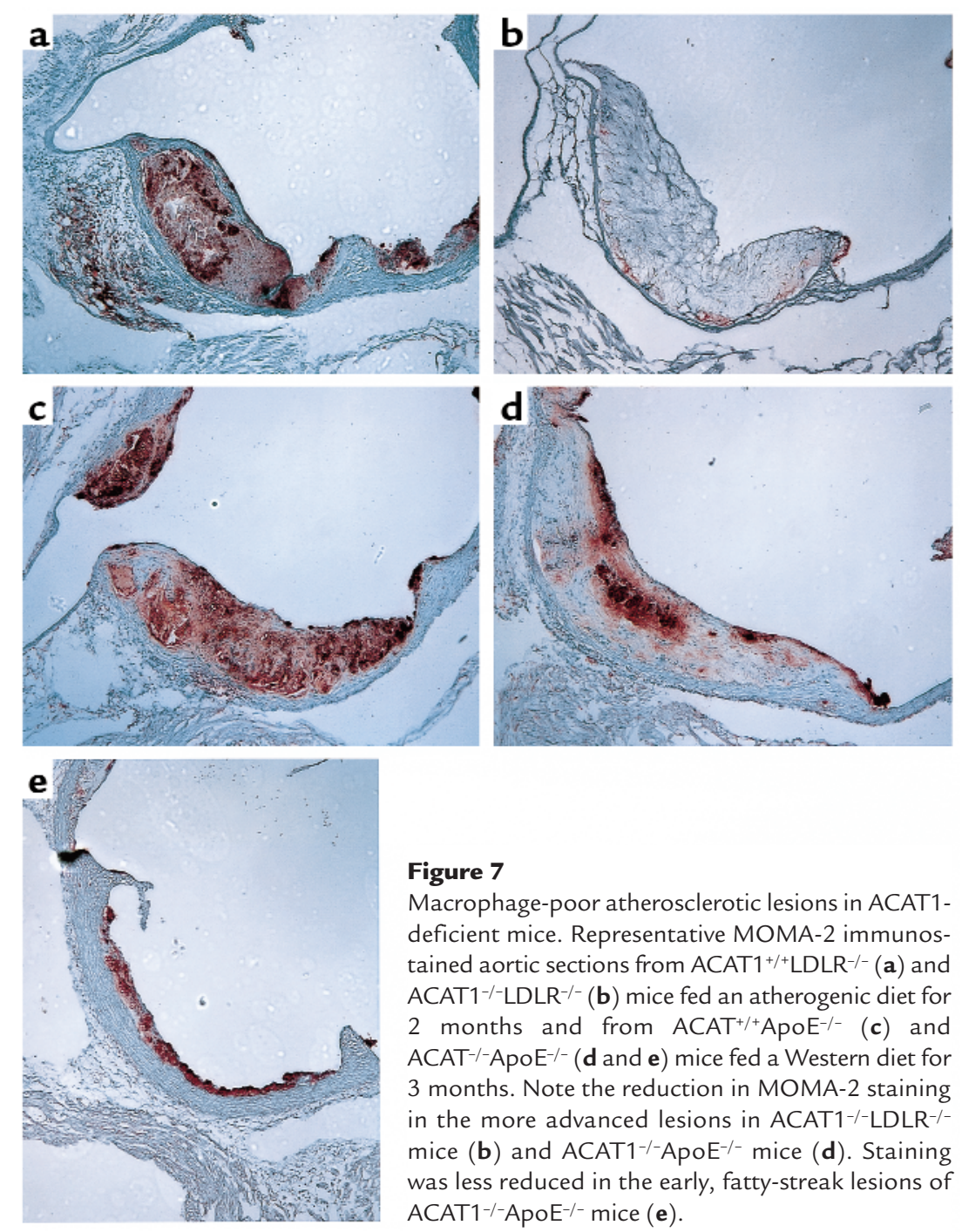

\section{Figure 7}

Macrophage-poor atherosclerotic lesions in ACAT1deficient mice. Representative MOMA-2 immunostained aortic sections from ACAT1 ${ }^{+/+} \mathrm{LDLR}^{-/-}(\mathbf{a})$ and ACAT1 1/-LDLR $/-$ (b) mice fed an atherogenic diet for 2 months and from $\mathrm{ACAT}^{+/+} \mathrm{ApoE}^{-/-}$(c) and $\mathrm{ACAT}^{-/-} \mathrm{ApoE}^{-/-}$(d and e) mice fed a Western diet for 3 months. Note the reduction in MOMA-2 staining in the more advanced lesions in ACAT1 $1^{-/-} \mathrm{LDLR}^{-/-}$ mice (b) and ACAT1-/-ApoE ${ }^{-/-}$mice (d). Staining was less reduced in the early, fatty-streak lesions of ACAT1 ${ }^{-/-}$ApoE $^{-/-}$mice (e). 
In vitro studies have shown that excess cholesterol-rich lipoproteins can be taken up by macrophages where ACAT converts the free cholesterol to cholesterol ester, a relatively inert compound, until the cholesterol load can be cleared through cholesterol efflux (4). However, if ACAT1 is absent, the accumulation of free cholesterol may be toxic to macrophages (30-32), causing cell death and, in the case of the skin, dermal deposition of cholesterol crystals. The cholesterol crystals are likely proinflammatory (33) and may stimulate macrophage recruitment, thereby perpetuating the cycle. The bone marrow transplantation experiment established that macrophages are a key cell type in the dermal xanthomatosis (rather than dermal fibroblasts, for example), as ACAT1-deficient macrophages were sufficient to cause skin lesions in LDLR-deficient mice. The decrease in serum cholesterol levels in the doubleknockout mice may have resulted from "clearance" by the skin, which acted as a cholesterol sink. Why the skin and the brain are the primary organs susceptible to cholesterol deposition is unclear but may reflect the existence of tissue-specific cholesterol clearance mechanisms that are overwhelmed when severe hyperlipidemia occurs in the setting of ACAT1 deficiency.

The loss of ACAT1 in macrophages did not prevent lesion development in the aortic root, and thus it appears that significant atherosclerotic lesions can develop in the absence of cholesterol ester-rich foam cells. Because neutral lipid staining was virtually absent in ACAT1-deficient lesions, it is unlikely that ACAT2 serves a significant compensatory role in this setting. Owing to the skin disease and relatively lower serum cholesterol levels in the ACAT1-deficient mice, we were unable to quantitate lesion size in these models. To circumvent this difficulty, in a separate study we are performing quantitative atherosclerosis studies in a bone marrow transplantation model in which ACAT1-deficient macrophages are transplanted into atherosclerosis-susceptible mice.

ACAT1 deficiency caused marked qualitative differences in atherosclerotic lesions, including a lack of cholesterol esters and markedly decreased macrophage content in advanced lesions. Because macrophage staining in ACAT1-deficient mice was present in early lesions, but virtually absent in advanced lesions, we presume that this finding reflects progressive cell death from free cholesterol toxicity, either through necrosis or apoptosis, as described previously in in vitro studies $(30-32)$. It is also possible that the reduction in macrophage staining represents an alteration in lesion macrophages such that the expression of the epitope normally recognized by the MOMA-2 antibody is altered. Despite the lack of macrophages in advanced lesions, ACAT1-deficient mice did develop lesions of substantial size. Because smooth muscle cell staining appeared to be similar in all groups, we presume that extracellular matter or undetected cell types contributed to the lesion area in ACAT1-deficient mice. The specific cellular and extracellular composition of
ACAT1-deficient lesions remains to be further determined, as does the clinical significance of these qualitative changes. Cholesterol ester-rich foam cells and their secreted products, such as matrix metalloproteinases, have been proposed as characteristics of areas of lesions that might be unstable and prone to plaque rupture (34). Thus, the altered lesion composition in ACAT1 deficiency may be associated with more stable lesions. This question was not directly addressed by our studies, however, and experimental models that test lesion stability are lacking.

In summary, ACAT1 deficiency in macrophages contributed to the development of extensive xanthomatosis in the setting of severe hyperlipidemia caused by apoE deficiency or LDLR deficiency. The lack of ACAT1 did not prevent atherosclerotic lesion development, although the lipid and cellular composition of lesions was markedly different from that in usual lesions. Clearly, the extensive tissue cholesterol deposition we observed occurred in the setting of total ACAT1 deficiency in models with marked alterations in cholesterol metabolism (equivalent to complete apoE deficiency or homozygous familial hypercholesterolemia, both of which are rare in humans). Partial inhibition of ACAT1, like what might be achieved by a pharmacological inhibitor, in the setting of less severe hyperlipidemia may not result in significant xanthomatosis. Nonetheless, our findings suggest that the development of specific and potent inhibitors of ACAT1 should be approached cautiously owing to concerns about possible deposition of cholesterol crystals in tissues such as skin and brain.

\section{Acknowledgments}

We thank E. Sande and L. Liu for technical assistance; D. Bunch for immunostaining of lesions; S. Turley for tissue cholesterol measurements; K. Feingold, P. Elias, and L. Nanney for assistance analyzing skin histology; J. Carroll and S. Gonzales for graphics; S. Ordway and G. Howard for editorial assistance; and B. Taylor for manuscript preparation. We also thank J. Herz, K. Weisgraber, and S. Young for helpful comments on the manuscript. This work was supported by a National Institutes of Health (NIH) training grant fellowship (to M. Accad); NIH grants HL57170 (to R. Farese), HL53989 and HL57986 (to M. Linton and S. Fazio), and AR41943 (to L. King); the Veterans Administration (grant to L. King); and The J. David Gladstone Institutes.

\footnotetext{
1. Suckling, K.E., and Stange, E.F. 1985. Role of acyl-CoA:cholesterol acyltransferase in cellular cholesterol metabolism. J. Lipid Res. 26:647-671.

2. Chang, T.Y., Chang, C.C.Y., and Cheng, D. 1997. Acyl-coenzyme A:cholesterol acyltransferase. Annu. Rev. Biochem. 66:613-638.

3. Chang, T.Y., Chang, C.C.Y., and Cadigan, K.M. 1994. The structure of acyl coenzyme A-cholesterol acyltransferase and its potential relevance to atherosclerosis. Trends Cardiovasc. Med. 4:223-230.

4. Brown, M.S., and Goldstein, J.L. 1983. Lipoprotein metabolism in the macrophage: implications for cholesterol deposition in atherosclerosis. Annu. Rev. Biochem. 52:223-261.

5. Sliskovic, D.R., and White, A.D. 1991. Therapeutic potential of ACAT inhibitors as lipid lowering and anti-atherosclerotic agents. Trends Pharmacol. Sci. 12:194-199.

6. Krause, B.R., and Bocan, T.M.A. 1995. ACAT inhibitors: physiologic
} 
mechanisms for hypolipidemic and anti-atherosclerotic activities in experimental animals. In Inflammation. Mediators and pathways. R.R. Ruffolo, Jr. and M.A. Hollinger, editors. CRC Press. Boca Raton, FL. 173-198.

7. Matsuda, K. 1994. ACAT inhibitors as antiatherosclerotic agents: compounds and mechanisms. Med. Res. Rev. 14:271-305.

8. Krause, B.R., Sliskovic, D.R., and Bocan, T.M.A. 1995. Emerging therapies in atherosclerosis. Expert Opinion on Investigational Drugs. 4:353-387.

9. Lee, H.T., et al. 1996. Inhibitors of acyl-CoA:cholesterol O-acyl transferase (ACAT) as hypocholesterolemic agents. CI-1011: an acyl sulfamate with unique cholesterol-lowering activity in animals fed noncholesterolsupplemented diets. J. Med. Chem. 39:5031-5034.

10. Wrenn, S.M., Jr., Parks, J.S., Immermann, F.W., and Rudel, L.L. 1995. ACAT inhibitors CL 283,546 and CL 283,796 reduce LDL cholesterol without affecting cholesterol absorption in African green monkeys. $J$. Lipid Res. 36:1199-1210.

11. Asami, Y., et al. 1998. HL-004, the ACAT inhibitor, prevents the progression of atherosclerosis in cholesterol-fed rabbits. Life Sci. 62:1055-1063.

12. Bocan, T.M.A., et al. 1991. Comparison of CI-976, an ACAT inhibitor, and selected lipid-lowering agents for antiatherosclerotic activity in iliac-femoral and thoracic aortic lesions. A biochemical, morphological, and morphometric evaluation. Arterioscler. Thromb. 11:1830-1843.

13. Matsuo, M., et al. 1995. Effect of FR145237, a novel ACAT inhibitor, on atherogenesis in cholesterol-fed and WHHL rabbits. Evidence for a direct effect on the arterial wall. Biochim. Biophys. Acta. 1259:254-260.

14. Nicolosi, R.J., Wilson, T.A., and Krause, B.R. 1998. The ACAT inhibitor, CI-1011 is effective in the prevention and regression of aortic fatty streak area in hamsters. Atherosclerosis. 137:77-85.

15. Chang, C.C.Y., Huh, H.Y., Cadigan, K.M., and Chang, T.Y. 1993. Molecular cloning and functional expression of human acyl-coenzyme A:cholesterol acyltransferase cDNA in mutant Chinese hamster ovary cells. $J$ Biol. Chem. 268:20747-20755.

16. Cases, S., et al. 1998. ACAT-2, a second mammalian acyl-CoA:cholesterol acyltransferase. Its cloning, expression, and characterization. J. Biol. Chem. 273:26755-26764.

17. Anderson, R.A., et al. 1998. Identification of a form of Acyl-CoA:cholesterol acyltransferase specific to liver and intestine in nonhuman primates. J. Biol. Chem. 273:26747-26754.

18. Oelkers, P., et al. 1998. Characterization of two human genes encoding acyl coenzyme A:cholesterol acyltransferase-related enzymes. J. Biol. Chem. 273:26765-26771

19. Meiner, V., et al. 1997. Tissue expression studies of mouse acyl CoA:cholesterol acyltransferase gene (Acact): findings supporting the existence of multiple cholesterol esterification enzymes in mice. J. Lipid Res.
38:1928-1933.

20. Miyazaki, A., et al. 1998. Expression of ACAT-1 protein in human atherosclerotic lesions and cultured human monocytes-macrophages. Arterioscler. Thromb. Vasc. Biol. 18:1568-1574.

21. Meiner, V.L., et al. 1996. Disruption of the acyl-CoA:cholesterol acyltransferase gene in mice: evidence suggesting multiple cholesterol esterification enzymes in mammals. Proc. Natl. Acad. Sci. USA. 93:14041-14046.

22. Piedrahita, J.A., et al. 1992. Generation of mice carrying a mutant apolipoprotein E gene inactivated by gene targeting in embryonic stem cells. Proc. Natl. Acad. Sci. USA. 89:4471-4475.

23. Gaw, A., Mancini, F.P., and Ishibashi, S. 1995. Rapid genotyping of low density lipoprotein receptor knockout mice using a polymerase chain reaction technique. Lab. Anim. 29:447-449.

24. Schwarz, M., Russell, D.W., Dietschy, J.M., and Turley, S.D. 1998. Marked reduction in bile acid synthesis in cholesterol $7 \alpha$-hydroxylase-deficient mice does not lead to diminished tissue cholesterol turnover or to hypercholesterolemia. J. Lipid Res. 39:1833-1843.

25. Fazio, S., et al. 1997. Leukocyte low density lipoprotein receptor (LDL$\mathrm{R})$ does not contribute to LDL clearance in vivo: bone marrow transplantation studies in the mouse. J. Lipid Res. 38:391-400.

26. Lindeskog, G.R., and Gustafson, A. 1972. Serum lipoprotein deficiency in diffuse "normolipemic" plane xanthoma. Arch. Dermatol. 106:529-532.

27. Williford, P.M., White, W.L., Jorizzo, J.L., and Greer, K. 1993. The spectrum of normolipemic plane xanthoma. Am. J. Dermatopathol. 15:572-575.

28. van Ree, J.H., et al. 1995. Atypical xanthomatosis in apolipoprotein Edeficient mice after cholesterol feeding. Atherosclerosis. 112:237-243.

29. Feingold, K.R., et al. 1995. Apolipoprotein E deficiency leads to cutaneous foam cell formation in mice. J. Invest. Dermatol. 104:246-250.

30. Warner, G.J., et al. 1995. Cell toxicity induced by inhibition of acyl coenzyme A:cholesterol acyltransferase and accumulation of unesterified cholesterol. J. Biol. Chem. 270:5772-5778.

31. Kellner-Weibel, G., et al. 1998. Effects of intracellular free cholesterol accumulation on macrophage viability. A model for foam cell death. Arterioscler. Thromb. Vasc. Biol. 18:423-431.

32. Tabas, I. 1997. Free cholesterol-induced cytotoxicity: a possible contributing factor to macrophage foam cell necrosis in advanced atherosclerotic lesions. Trends Cardiovasc. Med. 7:256-263.

33. Hasselbacher, P., and Hahn, J.L. 1980. Activation of the alternative pathway of complement by microcrystalline cholesterol. Atherosclerosis. 37:239-245.

34. Libby, P., et al. 1996. Macrophages and atherosclerotic plaque stability. Curr. Opin. Lipidol. 7:330-335. 